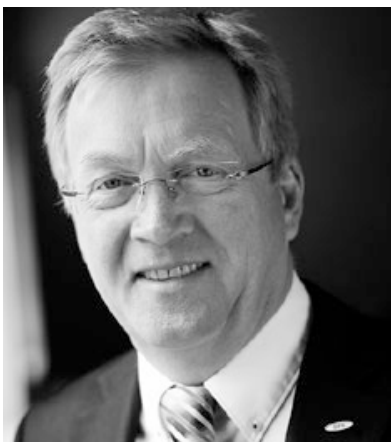

Andreas Westerfellhaus

Präsident des Deutschen Pflegerats (DPR)
Deutscher Pflegerat e.V.

Bundesarbeitsgemeinschaft

Pflege- und Hebammenwesen

In Kooperation mit

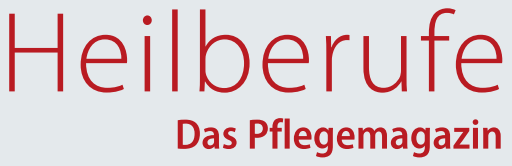

\title{
Editorial
}

\section{Gröhe setzt Expertenkommission ein}

D er Deutsche Pflegerat wurde von Bundesgesundheitsminister Hermann Gröhe in die Expertenkommission „Pflegepersonal im Krankenhaus" berufen. Das ist eine gute und wichtige Entscheidung. Aufgabe der Kommission ist es, sich mit der Frage einer sachgerechten Berücksichtigung des Pflegebedarfs im Vergütungssystem der Krankenhäuser zu befassen. „Eine angemessene Ausstattung mit Pflegepersonal ist sowohl für eine gute Versorgung der Patientinnen und Patienten als auch für die Arbeitssituation der Beschäftigten im Krankenhaus unabdingbar. Deshalb werden wir wichtige Maßnahmen ergreifen, damit Krankenhäuser mehr Pflegepersonal einstellen und dauerhaft beschäftigen können. Wir werden aber auch weitergehende Maßnahmen prüfen müssen, um die Pflege am Krankenbett zu stärken“, so der Minister.

Dem schließt sich der Deutsche Pflegerat an. Nicht vergessen werden darf dabei jedoch, dass bundesweit seitens der Krankenhausträger in den letzten Jahren rund 50.000 Vollzeitstellen im Pflegedienst abgebaut wurden. Ein solcher Personalabbau hat selbstverständlich bereits heute Auswirkungen auf die Qualität der Pflege und damit negative Auswirkungen auf die Patientensicherheit. Die Mitarbeiterinnen und Mitarbeiter der Pflege arbeiten an ihrem Limit. Wir benötigen somit auch Sofortmaßnahmen für die Entlastung der professionellen Pflege und zur Sicherstellung der Patientensicherheit. Dafür sind jährlich rund 2,5 Milliarden Euro an zusätzlichen Finanzmitteln für die Krankenhäuser nötig. Der aktuelle Gesetzentwurf sowie der Vorschlag der Bundestagsfraktionen von SPD und CDU/CSU von 500 Millionen Euro als Pflegezuschlag für Pflegepersonal sind davon nach wie vor weit entfernt.

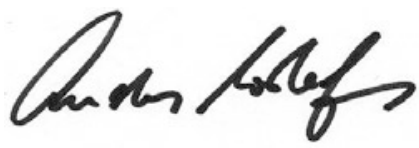

Andreas Westerfellhaus

Präsident des Deutschen Pflegerats (DPR)

Franz Wagner

Vize-Präsident des Deutschen Pflegerats (DPR) 